\title{
Interleukin-6 (-174 G/C) and Hepcidin HAMP (-582A/G) Gene Polymorphisms among Sudanese Patients with Anemia of Chronic Kidney Disease Treated with Erythropoietin
}

\author{
Khalid Mohamed Khalid Elhussain ${ }^{1,2,3, *}$, Amged Husssen Abdelrhman', Enaam Abdelrhman Abdelgadir ${ }^{4}$ \\ ${ }^{1}$ Department of Hematology and Immunohematology, Omdurman Islamic University, Sudan. \\ ${ }^{2}$ Omdurman Ahlia University, Faulty of Medicine, Sudan. \\ ${ }^{3}$ International University of Africa, Faculty of Medical Laboratory Sciences, Sudan. \\ ${ }^{4}$ Head Department of Pathology, Faculty of Medicine, Al-Neelain University, Sudan.
}

\begin{abstract}
How to cite this paper: Khalid Mohamed Khalid Elhussain, Amged Husssen Abdelrhman, Enaam Abdelrhman Abdelgadir. (2020) Interleukin-6 (-174 G/C) and Hepcidin HAMP (-582A/G) Gene Polymorphisms among Sudanese Patients with Anemia of Chronic Kidney Disease Treated with Erythropoietin. International Journal of Clinical and Experimental Medicine Research, 4(3), 34-40.

DOI: 10.26855/ijcemr.2020.07.001
\end{abstract}

Received: March 28, 2020

Accepted: April 30, 2020

Published: June 2, 2020

*Corresponding author: Khalid Mohamed Khalid Elhussain, Department of Hematology and Immunohematology, Omdurman Islamic University, Sudan; Omdurman Ahlia University, Faulty of Medicine, Sudan; International University of Africa, Faculty of Medical Laboratory Sciences, Sudan.

Email: klebs88@gmail.com

\begin{abstract}
Objectives. This is a case controls study used to determine the frequency of Interleukin-6 (-174 G/C) and hepcidin HAMP (-582A/G) gene polymorphisms among Sudanese patients with anemia of chronic kidney disease treated with erythropoietin by using the Polymerase Chain Reaction-Restriction Fragment Length Polymorphism (PCR/RFLP). Results. Significantly decreased levels in RBCS profile: RBCs count, Hb and PCVin patients with CKD and RBCS profile were observed. The polymorphisms (SNP) of the IL-6 gene and hepcidin (HAMP) gene promoter in Sudanese patients with ACKD were showed. the IL-6 (-174G/C) (SNP) was present in the hepcidin HAMP (-582A/G) (SNP) AA genotype 70 (35\%), AG 23 (11.5) and GG 7 (3.5\%) in 100 patients dialysis dependent and AA 166 (41.5\%), AG 34 (8.5\%) and GG 0 $(0 \%)$ in 200 control subjects, and the allele A are more frequent in patients affected by ACKD.
\end{abstract}

\section{Keywords}

Anemia, Interleukin-6 gene polymorphism, hepcidin HAMP gene polymorphism, Chronic kidney disease patients

\section{Introduction}

Anemia of chronic disease is anemia found in certain chronic disease state, is typically marked by the disturbance of iron homeostasis or hypoferremia [1]. This condition leads to shortage of iron for hemoglobin synthesis but the iron storage in bone morrow is left undisturbed. Anemia of chronic disease is the second most prevalent anemia after iron deficiency anemia, it can be triggered by wide range of inflammatory disorders such as infection, auto immune disease, chronic diseases aging process and malignancy, also known as anemia of inflammation [2, 3]. Accumulating data suggest that iron deficiency, is much more widespread and also affects patients with chronic kidney disease, especially those undergoing dialysis, and patients with congestive heart failure in whom iron deficiency impairs cardiovascular performance [4]. 


\subsection{Iron restriction}

First, systemic immune activation leads to weighty changes of iron trafficking, resulting in iron retention in macrophages and in decreased dietary iron absorption. Iron sequestration in macrophages is by far more important, because recycling of iron from senescent erythrocytes by macrophages accounts for $>90 \%$ of the daily iron demands for hemoglobin $(\mathrm{Hb})$ synthesis and erythropoiesis [5]. In laboratory animals and in humans, IL-6 motivates hepatocytes to produce hepcidin, the master regulator of iron homeostasis, predominantly through STAT3 [5]. Other cytokines, including IL-1 and activin B, can also stimulate hepcidin production, but their specific pathological role is less well established [6]. A kidney failure is a medical condition in which the kidneys fail to effectively filter waste products from the blood. Studies of hepcidin, an antimicrobial peptide that have a role as modulator of iron homeostasis has given a new insight for the management of anemia of chronic disease. Hepcidin, a peptide composed of 25 amino acid, is synthesized by hepatocyte [7], it inhibits iron release from macrophages, intestinal epithelial cells and from placental syncytiotrophoblasts by its interaction with the transmembrane iron exporter ferroportin [8]. Recent evidence indicates that, at higher concentrations, hepcidin may directly block iron export by occluding ferroportin [9]. A mechanism that may be especially important in limiting iron release from cells that lack endocytic machinery (erythrocytes) or in conditions under which endocytosis is slow, accelerating degradation of ferroportin mRNA increased production of hepcidin is induced by inflammatory via interleukin 6 (IL-6), hepcidin synthesis and secretion are controlled by proteins, HFE hemojuvelin and transferring receptor [10]. The human gene for IL-6 was cloned and reported by [11, 12, 13]. The functional promoter polymorphisms IL-6 C-174G and HAMPA-582G, which are the only SNPs that affect the IL-6 and hepcidin levels and associated with kidney function and CKD prevalence [14]. It was demonstrated that certain IL-6 genotypes may protect dialysis patients from cardiovascular events. In addition, various cytokines directly impact on duodenal or macrophage iron homeostasis. Tumor necrosis factor (TNF) reduces duodenal iron absorption by a poorly considered, but hepcidin-independent, mechanism. The cytokines IL-1, IL-6, IL-10, or TNF- $\alpha$ promote iron acquisition into macrophages through transferrin receptor-mediated endocytosis, via divalent metal transporter 1, or possibly also by increased iron acquisition by lactoferrin and lipocalin-2. 21 [15]. Recent evidence suggests that, during periods of enlarged erythrocyte destruction, erythrophagocytosis and iron recycling are primarily carried out by hepatic macrophages differentiating in the liver from circulating monocytes, rather than by local splenic macrophages [16]. The specific mechanisms by which iron restriction falls erythropoiesis have not been fully explained, but they involve active iron-regulated erythroid-specific mechanisms that decrease the synthesis of heme and $\mathrm{Hb}$, as well as hinder erythropoiesis, thereby protecting nonerythroid tissues from iron deficiency [17]. Heme concentration in erythrocyte precursors functions as a secondary iron-dependent manager of Hb synthesis and erythropoiesis [18]. Acting to promote improved iron supply during intensified erythropoiesis, Erythropoietinand hypoxia inhibit hepcidin formation via induction of hypoxia inducible factor 1, erythroferrone, matriptase-2, growth differentiation factor-15, or platelet derived growth factor-BB.14, 39-42Thus, the decreased availability and activity of Epo in iron deficiency negatively affect the induction of at least some of these hepcidin blockers, such as erythroferrone, thereby provoking hepcidin-mediated erythroid iron limitation43and impairing, via a vicious cycle, Epo signaling via Scribble [19].

\subsection{Decreased erythrocyte survival}

A shortened erythrocyte lifespan has been widely recognized in the inflammatory setting and has been attributed to improved erythrophagocytosis by hepatic and splenic macrophages caused by "bystander" deposition of antibody and complement on erythrocytes, mechanical damage from fibrin deposition in microvasculature, and stimulation of macrophages for increased erythrophagocytosis. Shortened erythrocyte survival is usually a minor factor in chronic AI; however, in acute infections, severe sepsis, or other critical illnesses accompanied by a high level of cytokine motivation, anemia is detected after hours or a few days (i.e., too rapidly to be accounted for by deficient erythropoiesis). It is reasonable that huge erythrophagocytosis, hemolysis, or pooling of erythrocytes, along with hemodilution, donate to this entity that awaits systematic scientific analysis [20]. Moreover, remediable iatrogenic factors are common in critical illness and include blood loss from phlebotomy and gastrointestinal blood loss caused by nasogastric tubes, anticoagulation, and the use of medications that promote gastroduodenal erosion or ulceration.

All these data suggest that significant genetic and epigenetic effects may modify inflammatory activity in CKD [21]. The present work was designed to study hepcidin HAMP (-582A/G) and IL-6 (-174G/C) gene polymorphisms and their possible association with anemia in Sudanese patients with chronic kidney disease. 


\section{Methods}

Study Participants: This a case controls study consisted of 300 individuals enrolled and divided into two groups: Control group: which included 200 apparently healthy volunteers of comparable age and socioeconomic status to the patients group without take any medications and intake the same nutrition with patients: 100 males (50\%) and 100 females (50\%) their mean age 33+_10.1 years and Patient group: which include 100 patients with anemia of chronic kidney disease treated by erythropoietin and exclude other types of anemia like sickle cell anemia, thalassemia, 57 males (57\%) and 43 females (43\%), their mean age 32+_10 years.

\subsection{Sample prepration and PCR detection of IL-6 and HAMP DNA Polymorphisms:}

Some of red blood cell profile (Hb, PCV, RBCs count and RBCs indices) were determined by the automated Hematology Analyzer and Cobas e 411 was used to determine the levels of serum iron, ferritin, TIBC, and transferrin saturation percentage. Molecular studies for detection of IL-6 (-174G/C) and HAMP (-582A/G) gene polymorphisms were done to all subjects using the Polymerase Chain Reaction/Restriction Fragment Length Polymorphism (PCR/RFLP) technique and included DNA extraction from peripheral blood leucocytes using a DNA extraction kit (NucleosSpin, Blood Quick Pure, Macherey-Nagel, Duren, Germany). Purity of extracted genomic DNA was detected by agarose gel electrophoresis of the yield on $1 \%$ agarose gel. In addition, the genomic DNA concentration was measured on a nano drop 1,000 spectrophotometer (Thermo-scientific -USA ) at 260 and $280 \mathrm{~nm}$. The mean concentration of the purified genomic DNA was 20.56ng/ul. After extraction, IL-6 Promoter region was amplified using published primer set: \{5-TTGTCAAGACATGCCAAAGTG3 (Forword) TCAGACATCTCCAGTCCTATA-3 (Reverse) $\}$ and HAMP gene promoter region also amplified using published primer set: \{5-GTACTCATCGGACTGTAGATGATTTAGC (forward), 5GTGACAGTCGCTTTTATGGGGC CT GC-3 (reverse). Amplification were done through Polymerase Chain Reaction (PCR) Cycle (95oc, 3min) x1, $\left(95^{0} \mathrm{c}, 30 \mathrm{~s}, 55^{\circ} \mathrm{c}, 30 \mathrm{~s}, 72^{\circ} \mathrm{c}, 60 \mathrm{~s}\right) \times 37$ : $\left(72^{\circ} \mathrm{c}, 10 \mathrm{~min}\right) \times 1$, using Dream Tag Green Master Mix Fermenats-Thermo, USA). Restriction digestion of PCR products was done using Fast Digest N1a III restriction enzyme (Fermentas-Thermo-USA) for IL-6 (-174G/C). The digestion products were subjected to $2 \%$ agarose gel electrophoresis and showed that allele $\mathrm{G}$ which did not contain the N1a III restriction enzyme site was digested to 230 bp and 54 bp fragments, whereas allele $C$ yielded 122 bp and 111 bp together with the 50 bp fragment (Figure 1). As regards the detection of HAMP (-582A/G) gene polymorphism, Fast Digest HindIII restriction enzyme (Fermentas-Thermo-USA) was used, the digestion products were subjected to $2.5 \%$ agarose gel electrophoresis and showed that allele A which did not contain the HindIII restriction enzyme site was digested to 200 bp fragments, whereas allele $\mathrm{G}$ yields $190 \mathrm{bp}$ and 90 bp fragments (Figure 2).

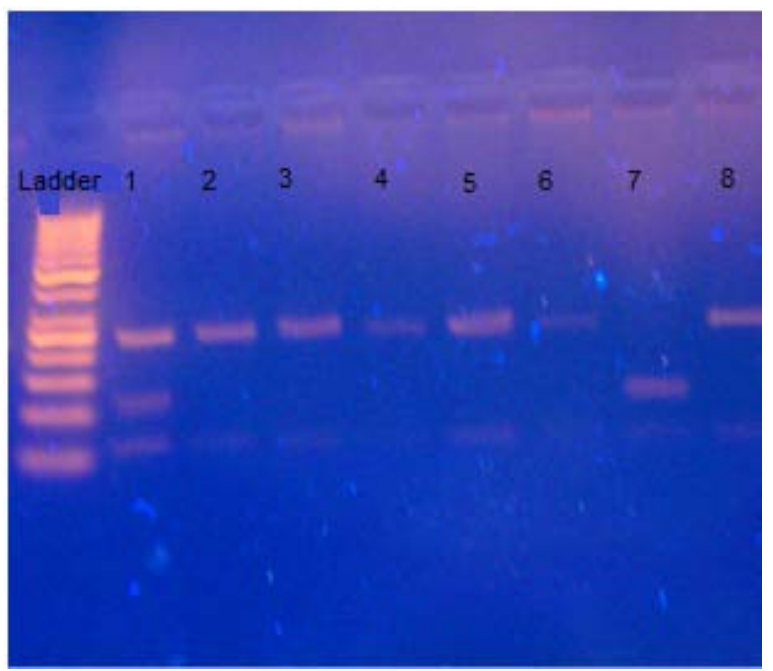

Figure 1. Agarose gel electrophoresis of PCR products digested with N1aIII restriction enzyme (IL-6).

50 bp DNA Ladder (50-1000 bp). Lane 1: GC heterozygote alleles (bands at 54 bp, 111 bp,122 bp and 233 bp). Lane 2, 3, 4, 5, 6, 8: GG homozygote alleles (bands at 54 bp and 233 bp). Lane 7: CC homozygote alleles (bands at 54 bp, 111 bp and 122 bp). 


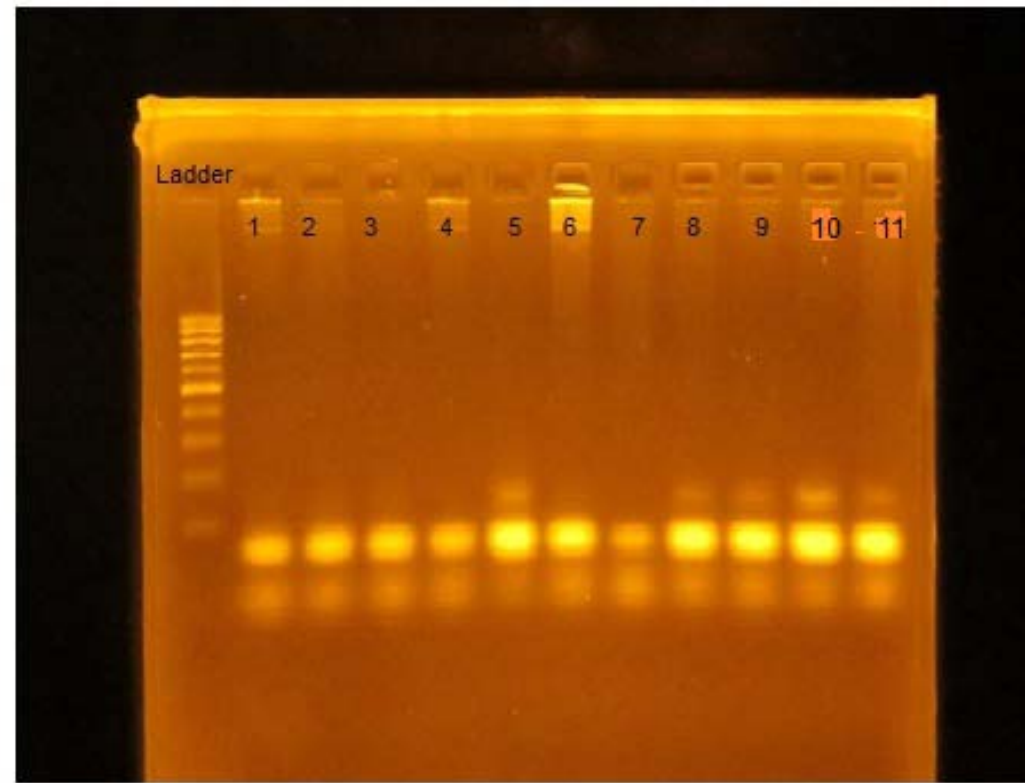

Figure 2. Agarose gel electrophoresis of PCR products digested with HindIII restriction enzyme (HAMP).

100 bp DNA Ladder (100- 1000 bp). Lane 1, 2, 3, 4, 6, 7: GG homozygote alleles (bands at 90 bp and190 bp). Lane 8, 9, 10, 11: AG heterozygote alleles (bands at $90 \mathrm{bp}, 190 \mathrm{bp}$, and $200 \mathrm{bp}$ ). Lane 5: AA homozygote alleles (band at $200 \mathrm{bp}$ ).

\section{Results}

Statistical significant differences were observed in the mean of red blood cell profile (RBCs count, Hb and PCV) between patients and control p-value $(0.000,0.000$, and 0.000$)$ respectively and not observed in the MCV, MCH and MCHC (Table 1). The mean value of the iron profile (Serum iron, s. ferritin and TS \%) between patients and control showed significant differences p-value $(0.000,0.000,0.000)$ respectively and not observed in TIBC (Table 1).

The distribution of genotypes and the allele frequencies to IL-6 (-174G/C) and HAMP(-582A/G ) in case and control are showed in Table 2 and Table 3. Genotypes were in Hardy-Weinberg equililibrium. The genotype distribution of the IL-6 (-174G/C) was GG 78, GC 18, CC 4 in 100 patients with ACKD and GG 32, GC 82, CC 2 in the 100 control subjects. The frequencies of the genotypes in patients with ACKD GG (52\%), GC (12\%), CC (2.6\%) was insignificant than control GG (56\%), GC (10.6\%), CC (2.3\%), P-value (0.142) (Table 2).

The genotype distribution of HAMP (-582A/G ) was AA 70, AG 23, GG 7 in patients with ACKD and AA 83, AG 17, GG 0, in 100 control subject, the frequencies of the genotypes in patients with ACKD AA ( 46.6\%) AG (15.3\%) GG ( 4.6\%) was insignificant than control AA ( 83\%), AG ( $17 \%$ ), GG ( 0\%) P-value 0.076. Evaluate whether the distribution of the IL-6 and HAMP genotypes was influenced by gender, male and female, same trend was observed in both men and women (P-value 0.747 and $\mathrm{P} 0.238$ respectively) (Table 3).

Table 1. Mean of Some Red Blood Cell Profile in 100 Patients with Anemia of Chronic Kidney Disease and 200 Control Group

\begin{tabular}{cccc}
\hline RBCs profile & $\begin{array}{c}\text { Mean } \pm \text { SD } \\
\text { Case }\end{array}$ & $\begin{array}{c}\text { Mean } \pm \text { SD } \\
\text { Control }\end{array}$ & P value \\
\hline RBCs count cell/l & $3.353 \pm 0.88$ & $4.048 \pm 0.47$ & $0.000^{\mathrm{S}}$ \\
HB g/dl & $10.62 \pm 2.4$ & $12.52 \pm 1.57$ & $0.000^{\mathrm{S}}$ \\
PCV \% & $32.59 \pm 6.82$ & $37.92 \pm 4.79$ & $0.000^{\mathrm{S}}$ \\
MCV fl & $93.09 \pm 10.12$ & $93.04 \pm 4.28$ & $0.974^{\mathrm{NS}}$ \\
MCH pg & $34.95 \pm 33.55$ & $30.59 \pm 1.42$ & $0.068^{\mathrm{NS}}$ \\
MCHC g/l & $316.34 \pm 37.58$ & $368.10 \pm 397.37$ & $0.070^{\mathrm{NS}}$ \\
\hline
\end{tabular}


Table 2. Genotype frequencies of interleukin-6 (-174G/C) gene polymorphism among studied groups

\begin{tabular}{|c|c|c|c|c|}
\hline \multirow{2}{*}{ Genotype } & \multicolumn{2}{|c|}{ Participants } & \multirow{2}{*}{ Total } & \multirow{2}{*}{$P$ value } \\
\hline & Case & Control & & \\
\hline GG & $78(52 \%)$ & 164(53.3\%) & (73.3\%) & \multirow{4}{*}{$0.142^{\mathrm{NS}}$} \\
\hline GC & $18(12 \%)$ & $32(10.6 \%)$ & (22.6\%) & \\
\hline $\mathrm{CC}$ & $4(2.6 \%)$ & $4(1.3 \%)$ & $(4 \%)$ & \\
\hline Total & $100(66.6 \%)$ & 200(67\%) & $300(100 \%)$ & \\
\hline Table 3. Genoty & of HAMP (- & polymorphis & died groups & \\
\hline \multirow{2}{*}{ Genotype } & \multicolumn{2}{|c|}{ Participants } & \multirow{2}{*}{ Total } & \multirow{2}{*}{$P$ value } \\
\hline & Case & Control & & \\
\hline AA & $70(46.6 \%)$ & 166(58.7\%) & (68.6\%) & \multirow{4}{*}{$0.076^{\mathrm{NS}}$} \\
\hline AG & $23(15.3 \%)$ & 34(11.3\%) & (26.6\%) & \\
\hline GG & $7(4.6 \%)$ & $0(0 \%)$ & $(4.6 \%)$ & \\
\hline Total & $100(66.7 \%)$ & $200(69.3 \%)$ & $300(100 \%)$ & \\
\hline
\end{tabular}

\section{Discussion}

CKD occur at all age, become more common with increasing age, after the age of 40 kidney filtration begins to fall by approximately $1 \%$ per year [21], according to the world health organization in the 2005, there were approximately 58 million deaths worldwide, with 35 million attributed to chronic disease, CKD frequency in Sudan $7 \%$ according to data presented by ministry of health and the prevalence of disease $10 \%$ (according to the 2010 Global Burden of Disease study) of the adult world population and its incidence is greater in men than women (3:1). Anemia is the most common blood abnormality observed in $15.4 \%$ in CKD stage 1-5 patients (NHANES).

Available data support of a G/C single nucleotide polymorphism (replacement of a nucleotide with another one) at the promoter 174 of the IL-6 gene in controlling of IL-6 gene transcription rate and consequently its circulating levels [22]. Two phenotypes for this polymorphism have been identified: the $174 \mathrm{G} / \mathrm{G}$ and $174 \mathrm{G} / \mathrm{C}$ genotypes as the high-producer phenotypes; and the $174 \mathrm{C} / \mathrm{C}$ genotypes as the low-producer phenotypes. The $174 \mathrm{G} / \mathrm{C}$ phenotype has been reported to be associated with the diseases with inflammatory background such as juvenile chronic arthiritis, Alzheimer disease, cardiovascular disease and chronic kidney disease. Hepcidin suppression causes the stored iron to be released by hepatocytes and macrophages while the intestinal absorption of iron increases. Hepcidin is elevated during inflammation and/or infection. Plasma levels of the IL-6 are influenced by the $-174 \mathrm{G} / \mathrm{C}$ polymorphism of the IL-6 promoterthat might to increase expression to more hepcidin. We studied the association between interleukin-6 (-174G/C) and hepcidin HAMP (-582A/G) gene polymorphisms and anemia of chronic kidney disease in Sudanese patients. No studies have been performed in order to test whether the G/C IL-6 gene and A/G HAMP gene polymorphisms associated with anemia of chronic kidney disease.

In this study, there is significant association between CKD and RBCs profile (RBCs count, Hb, PCV) and the type of anemia found is dimorphic normocytic normochromic and microcytic hypochromic anemia. Among these study group patients, the decreased levels of serum iron, TIBC and TS\% were found, however, serum ferritin levels were found to be elevated in this group. Findings consistent to this results agreement with a study on patients with CKD who reported that the serum ferritin levels are likely to be higher in ACKD patients due to inflammation. The situation in which the serum iron is low and the serum ferritin is high is frequently seen among ACKD patients. High ferritin levels may be observed in this disease because of functional iron deficiency or reticulo-endothelial blockade, was influenced by the age and duration of disease, we found positive correlation between the duration of disease and RBCS profile (RBCS count, Hb and PCV), serum iron and serum ferritin.

This study showed for the first time that the distribution and allele frequency for IL-6 (-174G/C) were compared in all subjects, no significant difference was observed among studied groups. In addition, no significant difference was observed in the frequency of IL-6 (-174G/C) alleles among studied groups P value 0.142.

The study showed also the genotype distribution and allele frequency for HAMP (-582A/G) were compared in all subjects, no significant difference was observed among studied groups. In addition, no significant difference 
was observed in the frequency of HAMP $(-582 \mathrm{~A} / \mathrm{G})$ alleles among studied groups $\mathrm{P}$ value 0.076 , conflicting results may be due to various reasons such as demographic features of subject and different life style, also sample size play a crucial role. This situation encourages more and more attempts to be made to further assess the associations of these polymorphisms with the disease.

\section{Limitations}

Further investigation are needed, our data support the hypothesis that the IL-6 G/C polymorphism and hepcidin HAMP (A/G) are important in the pathophysiology of ACKD.

List of abbreviations

\begin{tabular}{cc}
\hline ACKD & Anemia of Chronic Kidney Disease \\
A & Adenine \\
AI & Iron deficiency \\
CRP & C-Reactive Protein \\
C & Cytosine \\
DNA & Deoxyribonucleic Acid \\
Epo & Erythropoietin \\
G & Guanine \\
HAMP & Human Antimicrobial Peptide \\
HFE & High Iron Fe \\
IL-6 & Interleukin-6 \\
IL-1 & Interleukin-1 \\
SPSS & Social Packages Statistical \\
S & Significant \\
PCR-RFLP & Polymerase Chain Reaction-Restriction Fragment Length Polymorphism \\
TNF & Tissue Necrosis Factor \\
mRNA & Messenger Ribonucleic Acid \\
NS & Non Significant \\
\hline
\end{tabular}

\section{Declarations}

\section{Ethical approval and consent to participant}

Approval of this study was obtained from hematology department of medical laboratory science (MLS), Omdurman Islamic University, and Ministry of Health issued by the local ethical committee, Khartoum State, Sudan. Written consent was taken from each member of the study.

\section{Consent for publication}

Not applicable.

\section{Availability of data and materials}

The datasets generated during and/or analyzed in this study are not publicly available due to Bahri dialysis center ethical policy in order to protect participant confidentiality.

\section{Competing interest}

The authors declare that they have no competing interests.

\section{Funding}

No funding was obtained for this study. 


\section{Authors' contributions}

This work was carried out in collaboration among all authors. KMK and AH contributed in literature search and manuscript writing. KMK and AH had the main idea of the study and contributed in manuscript writing, KM contributed to clinic work, AH contributed in statistical analysis. KMK and EA supervised the study and critically reviewed the manuscript. All authors read and approved the final draft of the manuscript.

\section{Acknowledgment}

By the grace of Almighty Alla and his help, I completed the study, all praise to him, my gratitude goes to all authors, special thanks to the patients who were so cooperative and despite their pain.

\section{References}

[1] Hentze, M. W., Muckenthaler, M. U. \& Andrews, N. C. (2004). Balancing acts: molecular control of mammalian iron metabolism. Cell, 117, 285-297.

[2] Liao, G., Xiang, J., Huang, X. \& Yang, Y. (2012). A New “Mix-confined” Repeated Load Test for Evaluating Permanent Deformation of Asphalt Mixture. Journal of Testing and Evaluation, 40, 1177-1185.

[3] Roy, A., Kucukural, A. \& Zhang, Y. (2010). I-TASSER: a unified platform for automated protein structure and function prediction. Nature protocols, 5, 725.

[4] Tim Goodnough L., Comin-Colet J., Leal-Noval S., et al. (2017). Management of anemia in patients with congestive heart failure. Am J Hematol. 92(1): 88-93.

[5] Muckenthaler M. U., Rivella S., Hentze M. W., Galy B. (2017). A red carpet for iron metabolism. Cell. 168(3): 344-361.

[6] Canali S., Core A. B., Zumbrennen-Bullough K. B., et al. (2016). Activin B induces noncanonical SMAD1/5/8 signaling via BMP type i receptors in hepatocytes: evidence for a role in hepcidin induction by inflammation in male mice. Endocrinology. 157(3): 1146-1162.

[7] Nemeth, E., Tuttle, M. S., Powelson, J., Vaughn, M. B., Donovan, A., Ward, D. M., Ganz, T. \& Kaplan, J. (2004). Hepcidin regulates cellular iron efflux by binding to ferroportin and inducing its internalization. Science, 306, 2090-2093.

[8] Nemeth, E. \& Ganz, T. (2009). The role of hepcidin in iron metabolism. Acta haematologica, 122, 78-86.

[9] Aschemeyer S., Qiao B., Stefanova D., et al. (2018). Structure-function analysis of ferroportin defines the binding site and an alternative mechanism of action of hepcidin. Blood. 131(8): 899-910.

[10] Hunter, H. N., Fulton, D. B., Ganz, T. \& Vogel, H. J. (2002). The solution structure of human hepcidin, a peptide hormone with antimicrobial activity that is involved in iron uptake and hereditary hemochromatosis. Journal of Biological Chemistry, 277, 37597-37603.

[11] Taga, T., Hibi, M., Hirata, Y., Yamasaki, K., Yasukawa, K., Matsuda, T., Hirano, T. \& Kishimoto, T. (1989). Interleukin-6 triggers the association of its receptor with a possible signal transducer, gp130. Cell, 58, 573-581.

[12] Hirano, T., Akira, S., Taga, T. \& Kishimoto, T. (1990). Biological and clinical aspects of interleukin 6. Immunology today, 11, 443-449.

[13] Nishimoto, N. \& Kishimoto, T. (2006). Interleukin 6: from bench to bedside. Nature Reviews Rheumatology, 2, 619.

[14] Solak A. A., SöDERKVIST, B. K., MEDIN, C., HYLANDER, B. \& HEIWE, S. (2012). Health-related quality of life in different stages of chronic kidney disease and at initiation of dialysis treatment. Health and quality of life outcomes, 10, 71.

[15] Nairz M., Theurl I., Swirski F. K., Weiss G. (2017). "Pumping iron”-how macrophages handle iron at the systemic, microenvironmental, and cellular levels. Pflugers Arch., 469(3-4): 397-418.

[16] Theurl I., Hilgendorf I., Nairz M., et al. (2016). On-demand erythrocyte disposal and iron recycling requires transient macrophages in the liver. Nat Med. 22(8): 945-951.

[17] Khalil S., Delehanty L., Grado S, et al. (2018). Iron modulation of erythropoiesis is associated with Scribble-mediated control of the erythropoietin receptor. J Exp Med, 215(2): 661-679.

[18] Zhang S., Macias-Garcia A., Velazquez J., Paltrinieri E., Kaufman R. J., Chen J. J. (2018). HRI coordinates translation by eIF2 $\alpha \mathrm{P}$ and mTORC1 to mitigate ineffective erythropoiesis in mice during iron deficiency. Blood. 131(4): 450-461.

[19] Latour C, Wlodarczyk MF, Jung G, et al. (2017). Erythroferrone contributes to hepcidin repression in a mouse model of malarial anemia. Haematologica, 102(1): 60-68.

[20] Docherty A. B., Turgeon A. F., Walsh T. S. (2018). Best practice in critical care: anaemia in acute and critical illness. Transfus Med. 28(2): 181-189.

[21] Xu, J. Q., Mattock, M., Chusney, G. \& Burt, D. (1997). NIDDM as a disease of the innate immune system: association of acute-phase reactants and interleukin-6 with metabolic syndrome X. Diabetologia, 40, 1286.

[22] Travis, S., González-Quintela, A., Campos, J., Quinteiro, C., Domínguez, F. \& Loidi, L. (2010). Genetic study of the hepcidin gene (HAMP) promoter and functional analysis of the c.-582A> G variant. BMC genetics, 11, 110. 